\title{
Comparison between Analytical Solution and Experimental Setup of a Short Long Ytterbium Doped Fiber Laser
}

\author{
M. R. A. Moghaddam ${ }^{1 *}$, S. W. Harun ${ }^{1,2}$, H. Ahmad ${ }^{1}$ \\ ${ }^{1}$ Photonics Research Center, University of Malaya, Kuala Lumpur, Malaysia \\ ${ }^{2}$ Department of Electrical Engineering, University of Malaya, Kuala Lumpur, Malaysia \\ Email: *mramoghaddam@siswa.um.edu.my
}

Received April 10, 2012; revised May 6, 2012; accepted May 16, 2012

\begin{abstract}
In this research, Amplified Spontaneous Emission (ASE) spectrum characteristics for a highly $\mathrm{Yb}^{3+}$ doped glass fiber with different pump powers and pump wavelengths are scrutinized. ASE spectral profile and wavelength shift corresponding to different optical fiber lengths are measured. Highly-doped $\mathrm{Yb}^{3+}$ fiber lasers in a linear-cavity are both experimentally and analytically investigated. Rate equations are solved using quasi-numerical models. Numerical results are reported for wide range of operating conditions to enable design optimization. The model takes into account the scattering loss and the distributed laser loss power density in strongly pump condition. The effects of various parameters such as pump power, pump wavelength, signal wavelength and fiber length on the output power and laser threshold are studied. Theoretical results are shown to be in good agreement with the experimental data.
\end{abstract}

Keywords: Ytterbium Doped Fiber; ASE; Rate Equation; Runge-Kutta

\section{Introduction}

Fiber lasers have many advantages such as high conversion efficiency, immunity from thermal lensing effect, simplicity of optical construction and excellent beam quality. Ytterbium doping is attractive for high-power fiber lasers because of its high efficiency and strong pump absorption. Ytterbium-doped silica fibers exhibit very broad absorption and emission bands, from $800 \mathrm{~nm}$ to $1064 \mathrm{~nm}$ for absorption and $970 \mathrm{~nm}$ to $1200 \mathrm{~nm}$ for emission $[1,2]$. The simplicity of the level structure provides freedom from unwanted processes such as excited state absorption, multi-phonon nonradiative decay, and concentration quenching [3,4].

Much work on Ytterbium Doped Fiber lasers (YDFLs) focuses on increasing the efficiency of the Laser and some authors have studied Fiber lasers theoretically. In our earlier work a laser action was demonstrated with near $90 \%$ slope efficiency using a fabricated YDF as a gain medium [5].

In recent years, the output powers from doped fiber lasers and amplifiers have been scaled and one approach for further power scaling these light sources is to increase the concentration to reduce the required fiber length and avoid nonlinear effects.

${ }^{*}$ Corresponding author.
However, pumping doped silica fiber with high concentrations can result in excess loss at the pump and signal wavelengths owing to photodarkening, which can significantly reduce the overall conversion efficiency and degrade the long-term performance [6].

In this article, we theoretically and experimentally analyze a YDF which is useful for low noise systems and very short application lengths. Rate equations are solved using semi-numerical models $[7,8]$. This model takes into account the scattering loss and the distributed laser loss power density in strongly pump condition [9]. The effects of various parameters such as pump power, pump wavelength, signal wavelength and fiber length on the output power and laser threshold are studied.

\section{Experimental Setup}

The experimental setup used for the lasing experiments is illustrated in Figure 1. In the work, different lengths $(L)$ of YDF are pumped through a WDM by pigtailed diode lasers.

The YDF used is a heavily-doped Ytterbium silica fiber with a core absorption coefficient $\left(\alpha_{0}\right)$ of $1200 \mathrm{~dB} / \mathrm{m}$ at $976 \mathrm{~nm}$.This fiber has a core/cladding diameter of $4 / 125 \mu \mathrm{m}$, a numerical aperture of 0.2 and a cut-off wavelength of $1010 \mathrm{~nm}$. The photodarkening effect in 


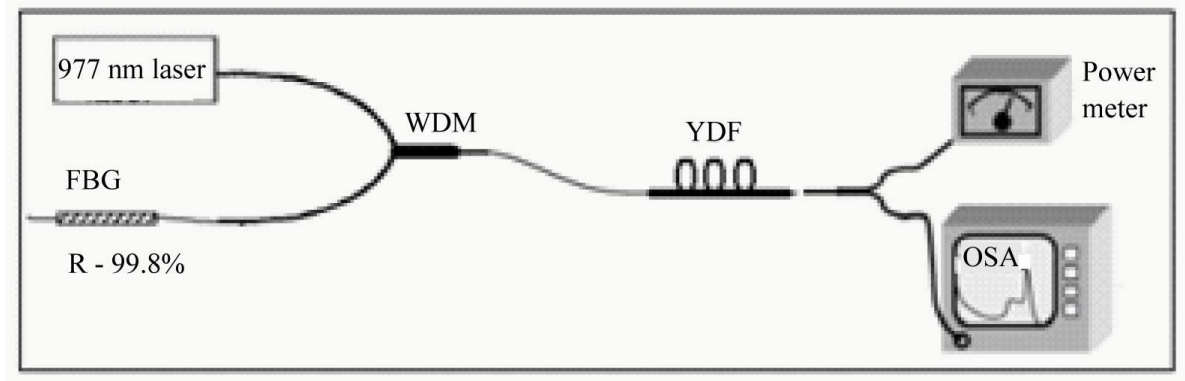

Figure 1. Experimental setup of laser cavity.

this fiber has been reduced by technique of direct nanoparticle deposition [10].

The output characteristics of the laser are measured using a power meter and an optical spectrum analyzer (OSA).

The reflectivity of output coupler is varied from $4 \%$ to $95 \%$. For a lossless fiber laser, the output power is almost constant over reflectivity range since the higher reflectivity increases the power inside the cavity and simultaneously decreases a relative output power. For a lossy system, for example in a resonator with an imperfect back reflector or in an unmatched system with different numerical apertures, the higher output reflectivity increases a propagation length of the signal due to more round trips and it results in a higher loss. Therefore, the output power increases with the transmission of the output coupler.

In this work, the resonator cavity is formed between a Fiber Bragg Grating (FBG) and a perpendicularly cleaved YDF end. The experiments are carried out for two different FBGs having a peak reflectivity of about $95 \%$ with the peak wavelengths of $1064.94 \mathrm{~nm}$, and $1028.02 \mathrm{~nm}$. We compared the laser output characteristics for these two signal wavelengths. The forward and backward amplified spontaneous emission (ASE) spectrum at different pump wavelengths and power levels are also compared.

\subsection{Comparison of ASE Spectrum}

The ASE spectra of a forward $975 \mathrm{~nm}$ pumping scheme for five different lengths between 10 to $400 \mathrm{~cm}$ are shown in Figure 2. This figure shows that for a given pump power $(200 \mathrm{~mW})$, the wavelength corresponding to peak of ASE varies from 1055.42 to $1074 \mathrm{~nm}$ by increasing the YDF length from 10 to $400 \mathrm{~cm}$. In other words there is an overall shifting of the ASE spectrum toward the higher wavelength side with increasing the length of the fiber. Meanwhile, the FWHM of the observed spectra decreases from $48 \mathrm{~nm}$ to $33 \mathrm{~nm}$ as the YDF length is increased from 10 to $400 \mathrm{~cm}$ as shown in Figure 3.

In fact, for longer fibers the ASE would be reabsorbed in the fiber as it propagates beyond the point where the pump is attenuated. Since the absorption cross-section is higher on the lower wavelength side, the re-absorption is more prominent in this region.

From residual transmitted pump intensity, it is found that the pump power is completely absorbed even in a short piece of this fiber. Inset figure verifies that the shape of the ASE spectra don't show any considerable change with the pump power.

For a given fiber length, the experimental results show that the ASE (fluorescence) bandwidth can be further increased as the pumping wavelength decreases. From the result obtained for $100 \mathrm{~mW}, 975 \mathrm{~nm}$ forward pumping, an ASE bandwidth of $32 \mathrm{~nm}$ is obtained for $4 \mathrm{~m}$ long YDF. While under the same condition, the ASE bandwidth is measured to be $55 \mathrm{~nm}$ at a shorter pumping wavelength $(967 \mathrm{~nm})$. However the ASE bandwidth decreases from $55 \mathrm{~nm}$ to $28 \mathrm{~nm}$ as the pump power is increased from 100 to $600 \mathrm{~mW}$.

A comparison of the ASE peak position for two different pumping wavelengths in a forward pumping scheme having a piece of $4 \mathrm{~m}$ long YDF is shown in Figure 4. From the figure, we can deduce that moving toward longer pump wavelength results in shifting ASE peak toward longer wavelength.

As shown in Figure 5, the backward ASE does not suffer the ASE re-absorption. Therefore it shows a wider bandwidth in the figure inset.

\subsection{Laser Setup}

In a lossless fiber laser, the output power does not change with the fiber length. However, in reality, an increase in the fiber length makes a larger absorption and a larger signal loss. Figure 6 shows the 1064 nm laser output power versus $975 \mathrm{~nm}$ launched pump power. The output power is compared in this figure for different fiber lengths between 0.3 to $2 \mathrm{~m}$.

Using a numerical aperture (NA) of 0.2 for YDF used and a NA of 0.14 for FBG in the setup, the maximum output power is measured to be $110 \mathrm{~mW}$ for $2 \mathrm{~m}$ of YDF at maximum pump power. As also shown in the figure lasing starts at a different threshold power depending on the YDF length. 


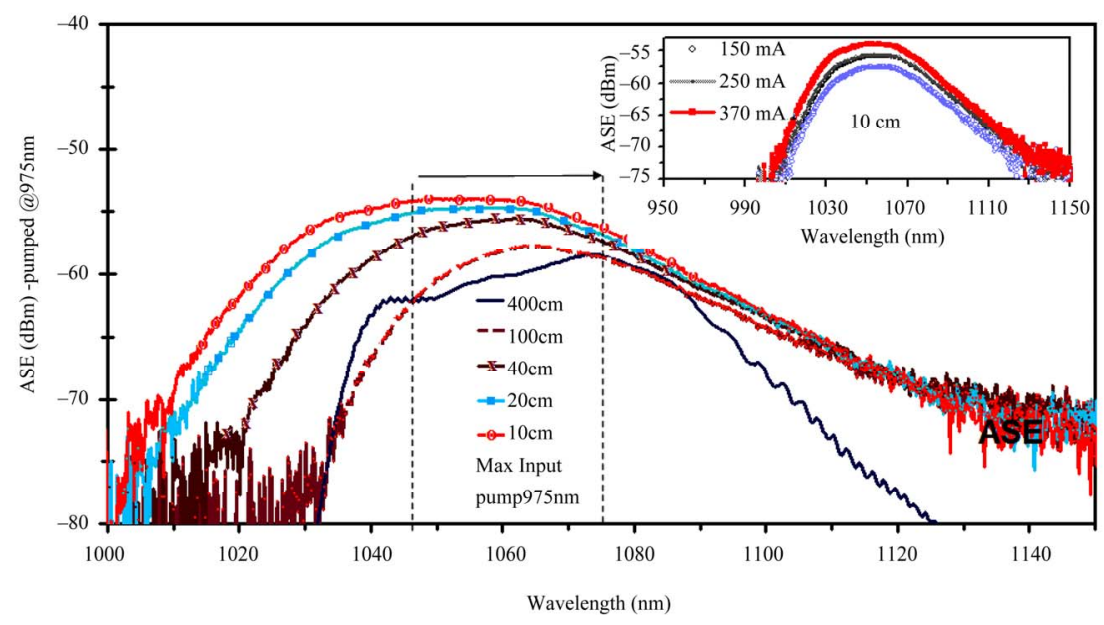

Figure 2. ASE spectrum shift for different fiber lengths and input powers.

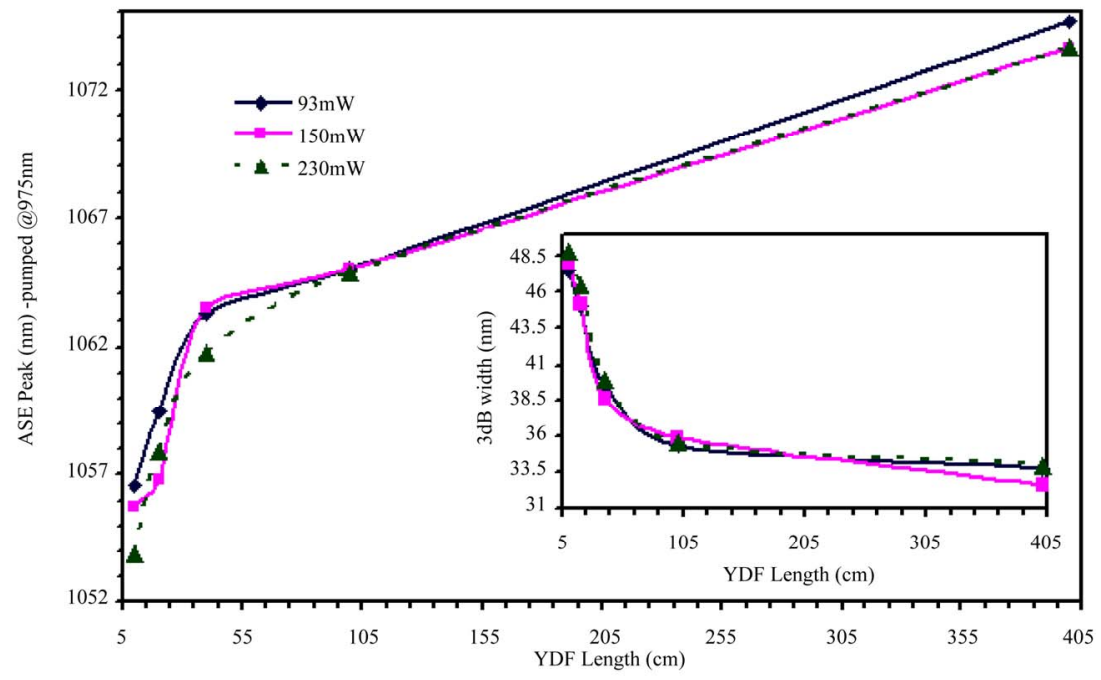

Figure 3. The variation ASE peak and bandwidth under $975 \mathrm{~nm}$ forward pumping for different fiber lengths.

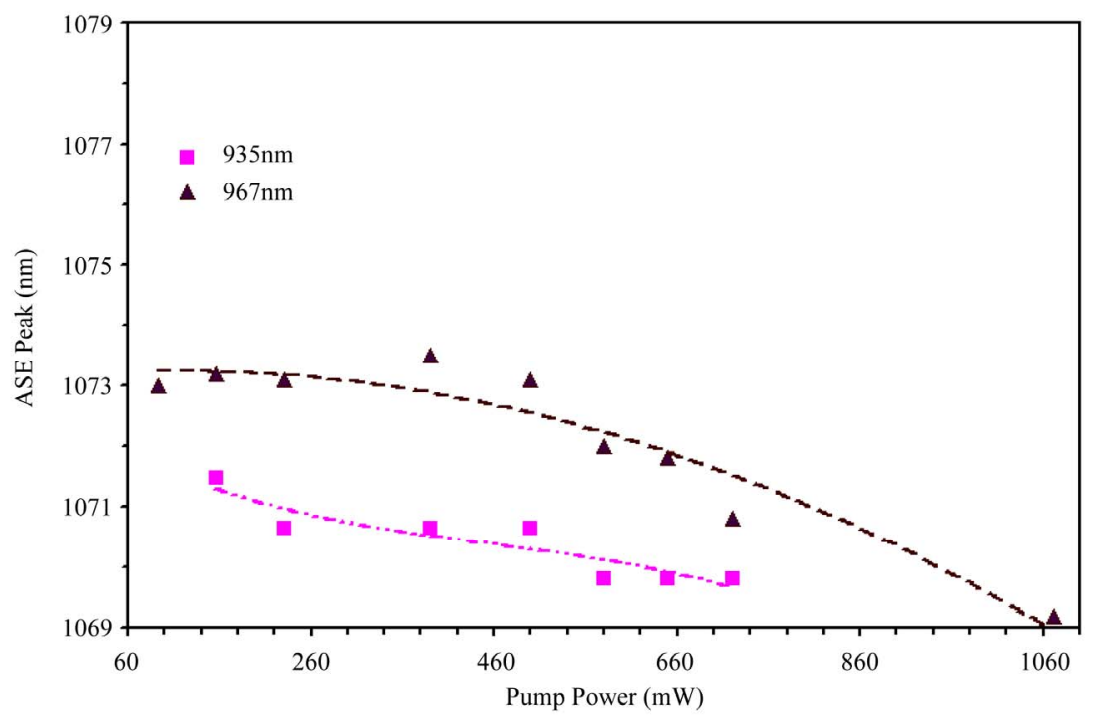

Figure 4. The variation of ASE peak position with pumping wavelength. 


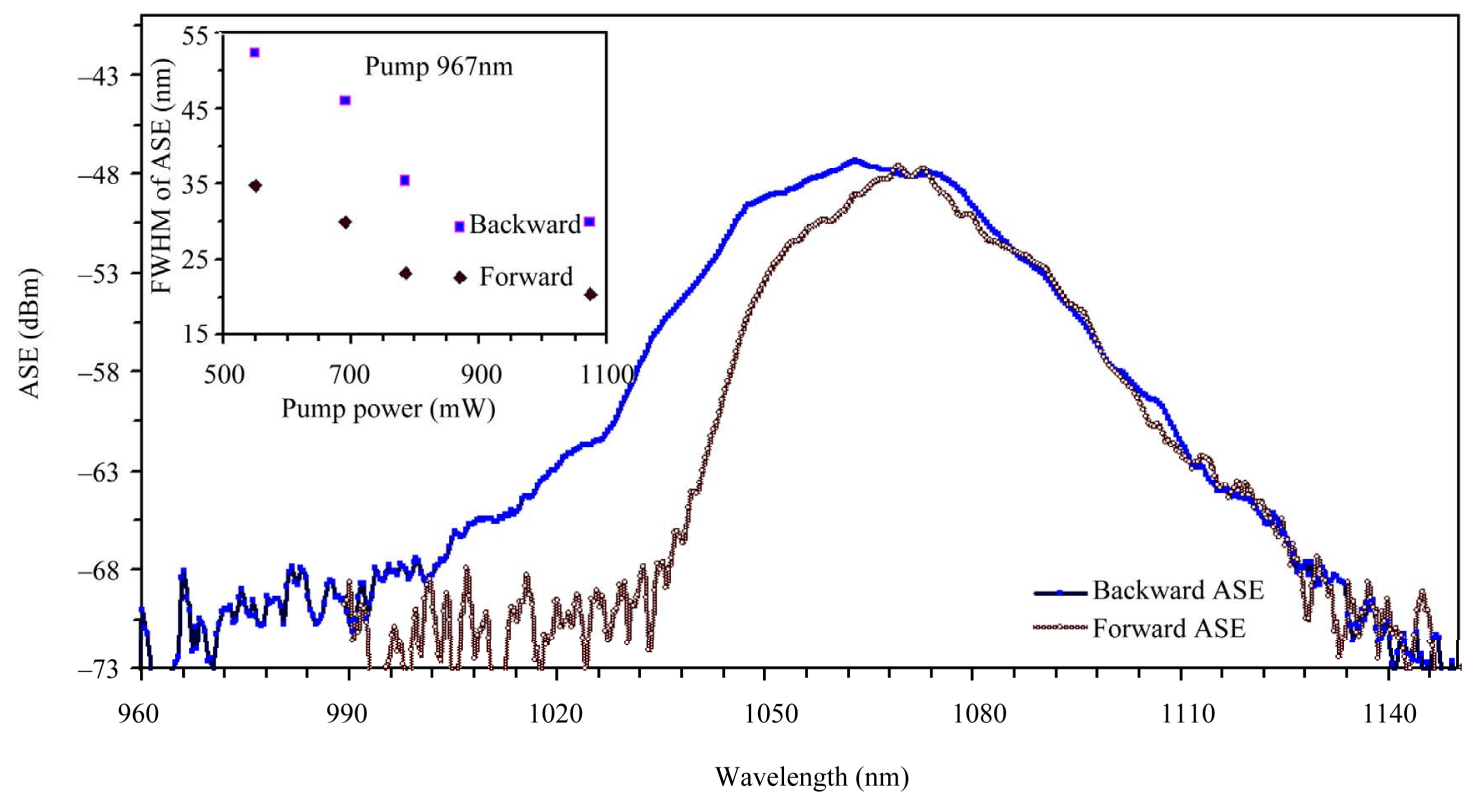

Figure 5. Comparison of ASE in forward and backward pumping schemes.

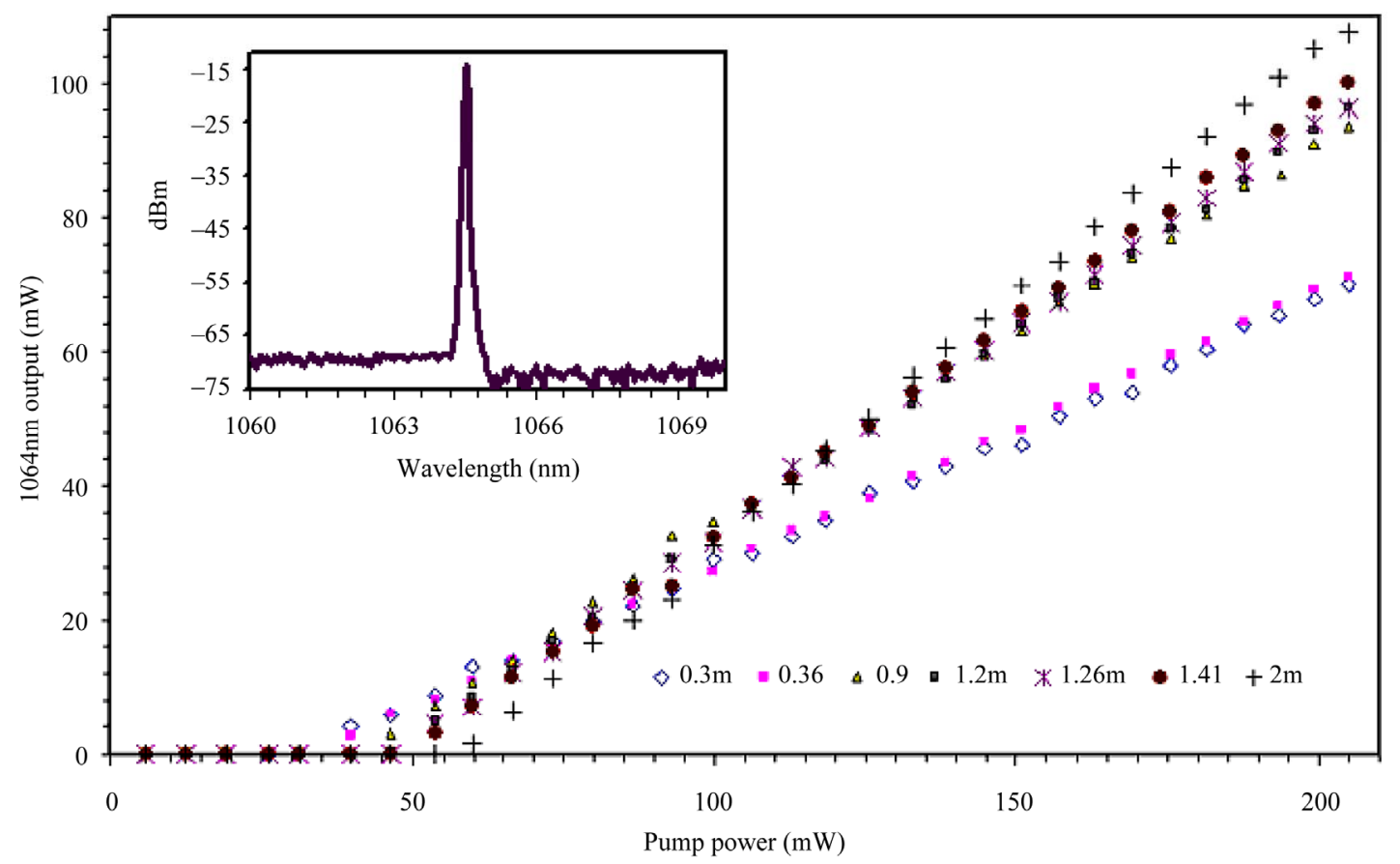

Figure 6. Output power as a function of the $975 \mathrm{~nm}$ pump power for $1064 \mathrm{~nm}$ wavelength. Inset shows typical laser spectrum.

On the other hand, for shorter operating wavelength the output power is optimized at a shorter YDF length near $30 \mathrm{~cm}$. Figure 7 shows the the output power of the laser versus pump power when the operating wavelength $(\lambda)$ is $1028.02 \mathrm{~nm}$. This figure indicates that a long YDF does not have the higher slope efficiency and shows that the laser threshold decreases as the length of YDF decreases. It is also found that the bandwidth of laser line $(0.08 \mathrm{~nm})$ remains unchanged with the pump power.

\section{Rate Equations}

The spectroscopy of the $\mathrm{Yb}$ ion is simple as compared to other rare-earth ions. Nonetheless, when Ytterbium is doped in the amorphous silica glass, the emission and absorption spectrum broaden beyond that which would be found in a crystalline structure due to the sub-bands of the two manifolds, ${ }^{2} \mathrm{~F}_{5 / 2}$ and ${ }^{2} \mathrm{~F}_{7 / 2}$ [11,12]. Figure 8 shows the absorption and emission cross sections for YDF used in this work [13]. The three levels 


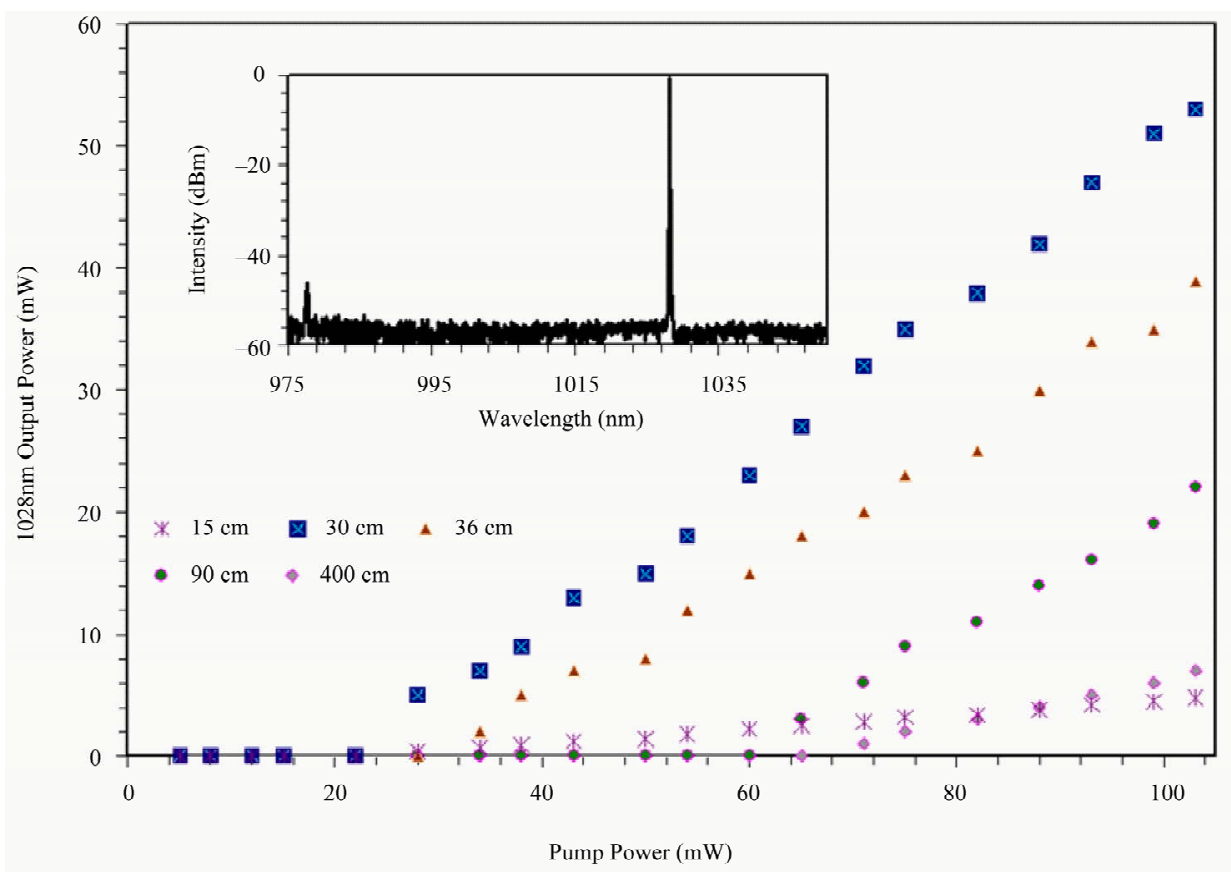

Figure 7. Output power as a function of the pump power for operating at $1028 \mathbf{~ n m}$.

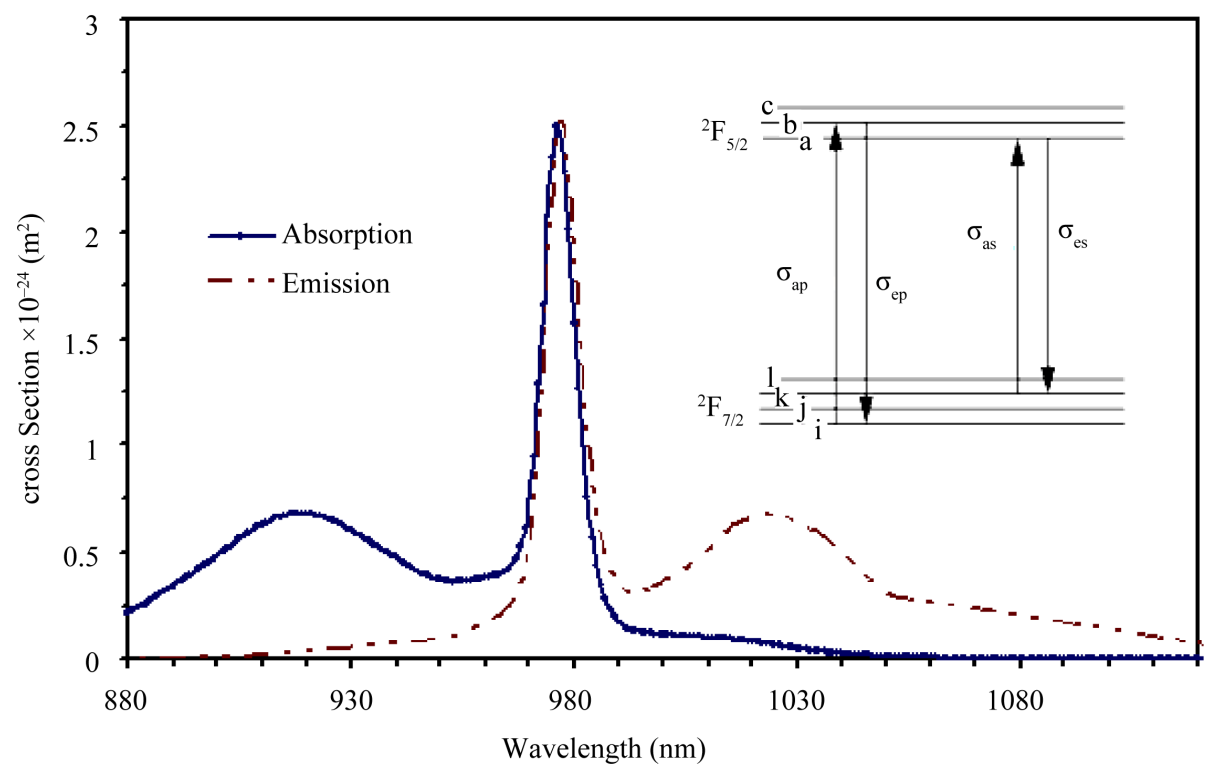

Figure 8. Absorption (solid line) and emission (dashed line) cross-sections used for numerical calculations. Inset-The energy levels structure of $\mathbf{Y b}^{3+}$ ions in silica.

in the upper manifold $\left({ }^{2} \mathrm{~F}_{5 / 2}\right)$ are denoted by a, b, and c in the inset of the figure.

The analytical solutions of the rate equations in the pre- sence of optical scattering loss and strongly pumped condition has been reported in [14]. The set of coupled equations and boundary conditions to be solved are given as:

$$
\frac{N_{2}(z)}{N}=\frac{\frac{\Gamma_{p} \sigma_{a p}\left[P_{p}^{+}(z)+P_{p}^{-}(z)\right]}{h v_{p} A}+\frac{\Gamma_{s} \sigma_{a s}\left[P_{s}^{+}(z)+P_{s}^{-}(z)\right]}{h v_{p} A}}{\frac{\Gamma_{p}\left(\sigma_{a p}+\sigma_{e p}\right)\left[P_{p}^{+}(z)+P_{p}^{-}(z)\right]}{h v_{p} A}+\frac{1}{\tau}+\frac{\Gamma_{s}\left(\sigma_{a s}+\sigma_{e s}\right)\left[P_{s}^{+}(z)+P_{s}^{-}(z)\right]}{h v_{p} A}}
$$




$$
\begin{aligned}
& \pm \frac{\mathrm{d} P_{p}^{ \pm}(z)}{\mathrm{d} z} \\
& =-\Gamma_{p}\left[\sigma_{a p} N-\left(\sigma_{a p}+\sigma_{e p}\right) N_{2}(z)\right] P_{p}^{ \pm}(z)-\alpha_{p} P_{p}^{ \pm}(z) \\
& \pm \frac{\mathrm{d} P_{s}^{ \pm}(z)}{\mathrm{d} z} \\
& =\Gamma_{s}\left[\left(\sigma_{a s}+\sigma_{e s}\right) N_{2}(z)-\sigma_{a s} N\right] P_{s}^{ \pm}(z)-\alpha_{s} P_{s}^{ \pm}(z)
\end{aligned}
$$

where $\tau$ ascertains the spontaneous lifetime, and $\alpha_{P}\left(\alpha_{S}\right)$ denotes scattering loss coefficient of pump (oscillating) light which is independent of $z$. Moreover $\sigma_{a p}\left(\sigma_{a s}\right)$ and $\sigma_{e p}\left(\sigma_{e s}\right)$ are absorption and emission cross sections of the pump (laser) light, respectively (see Figure 8).

Equation (1) describes the distribution of the upper level population $N_{2}$ at position $z$ along the YDF, while the evolution of the co propagating $\left(P_{P}^{+}(z)\right)$ and counter propagating $\left(P_{P}^{-}(z)\right)$ pump powers and laser powers (are given by Equations (2) and (3). It is worth to point out that the concentration density of $\mathrm{Yb}^{3+}$ ions $N=$ $N_{1}(z)+N_{2}(z)$ are set to a constant value and the strongly pumped condition is used based on

$N_{2}(z) \ll N$ and $\sigma_{a s} \ll \sigma_{e s}$.

In the above equations, $h, c, v_{p}$ and $v_{S}$ denote Planck's constant, the speed of light in vacuum, the pump and oscillation frequencies respectively. Some of the important parameters used in the numerical calculations and the procedure to obtain these parameters have been reported in [15]. In the modeling the active fiber with a length of $L$ and a core area of $A=1.28 \times 10^{-11} \mathrm{~m}^{2}$ has an average constant concentration per unit volume $(N)$ of $1.02 \times 10^{26} \mathrm{ions} / \mathrm{m}^{3}$. The excited state lifetime, the background loss at $1000 \mathrm{~nm}, \Gamma_{P}$ and $\Gamma_{S}$ (power filling factors) are also set at $0.88 \mathrm{~ms}, 40.99 \mathrm{~dB} / \mathrm{km}, 0.7$ and 0.9 respectively. Where $\Gamma_{S}$ represents the contribution of laser power in the core and $\Gamma_{P}$ illustrates the fraction of the pump power actually coupled to the core.

According to the Figure 9, the pump power is injected at $z=L$ or 0 at a known level and the laser beam is generated and propagated along the fiber. At the ends of the YDF, a portion of the power is ultimately reflected. Hence the rate equations are to be solved subject to the boundary conditions [7]:

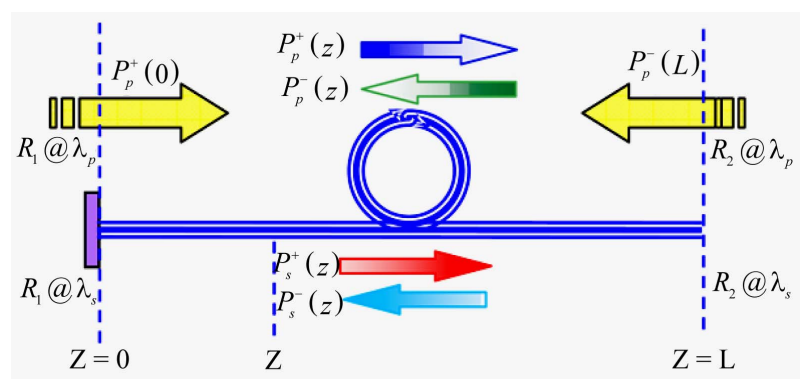

Figure 9. Schematic illustration of end-pumped YDFL.

$$
\begin{gathered}
P_{s}^{+}(0)=R_{1} P_{s}^{-}(0) \\
P_{s}^{-}(L)=R_{2} P_{s}^{+}(L)
\end{gathered}
$$

where $R_{2}(\lambda)$ and $R_{1}(\lambda)$ are the reflectivities of $\lambda$ and $P_{S}^{ \pm}(L)$ and $P_{S}^{ \pm}(0)$ are the oscillating powers at $z=L$ and 0 respectively. In numerical methods, the initial values of $P_{S}^{ \pm}(0)$ or $P_{S}^{ \pm}(L)$ would be determined using shooting methods [8].

In this work, Equations (1)-(3) are solved without neglecting the scattering loss and approximating in the distributed loss laser power density [7]. The calculations are run numerically in fourth-order Runge-Kutta method by using Matlab.

\section{Simulation Results and Discussion}

Figure 10(a) compares the numerical and experimental results of threshold power as a function of the fiber length for operating wavelengths of $1028 \mathrm{~nm}$ and 1064 $\mathrm{nm}$. The numerical and experimental output powers as a function of the fiber length are also compared for operating wavelengths of $1028 \mathrm{~nm}$ and $1064 \mathrm{~nm}$ as shown in Figure 10(b). In the figures, forward pumping power is fixed at $93 \mathrm{~mW}$.

As demonstrated in Figure $\mathbf{1 1}$ for a $2 \mathrm{~m}$ long YDF and pumping at $974 \mathrm{~nm}$, the minimum of the threshold power is obtained at operating wavelength of $1080 \mathrm{~nm}$.

For a given length of the resonator (e.g. $2 \mathrm{~m}$ long YDF), by choice of an appropriate pumping and operating wavelength, the threshold power can be reduced by more than six times as shown in Figure 12.

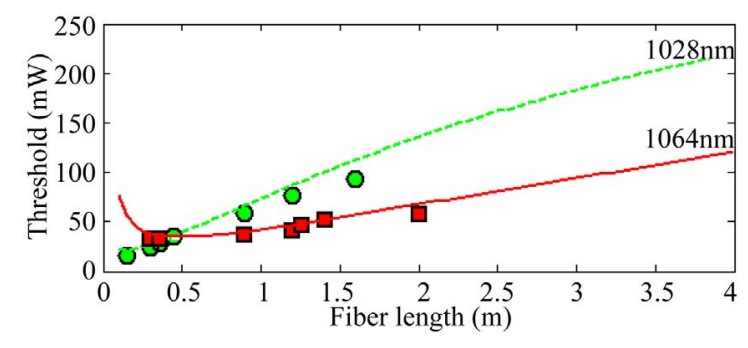

(a)

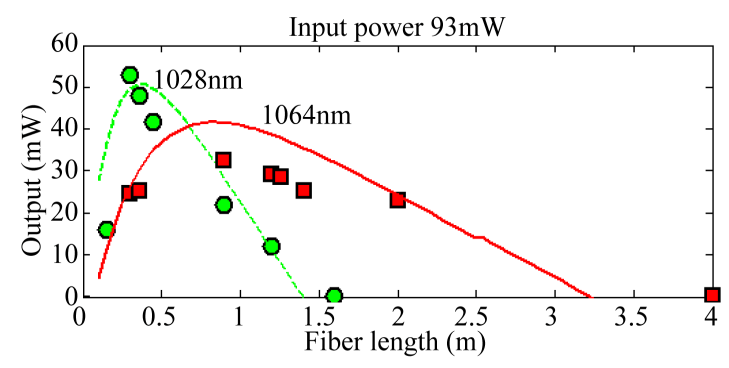

(b)

Figure 10. (a) Threshold power and (b) output power against fiber length at $93 \mathrm{~mW}$ of input pump power. 


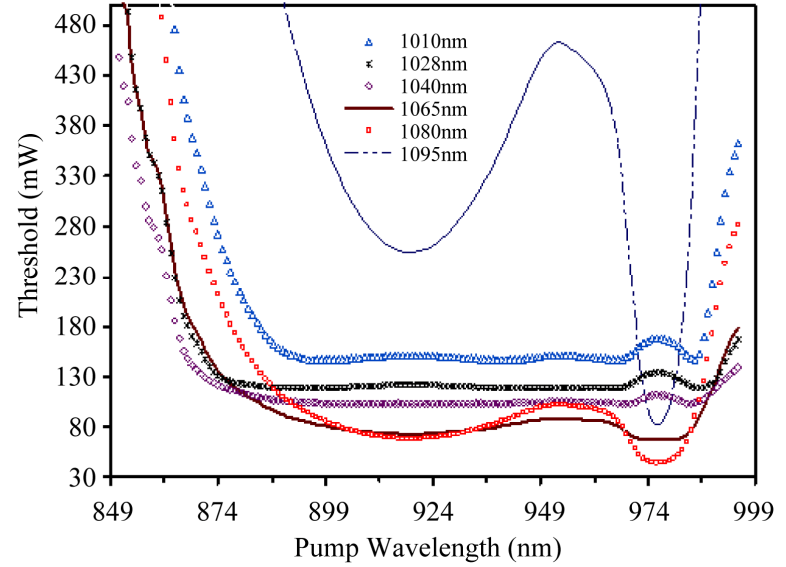

Figure 11. Laser threshold as a function of pumping wavelength for various operating wavelengths. The YDF length is fixed at $\mathbf{2} \mathbf{m}$.

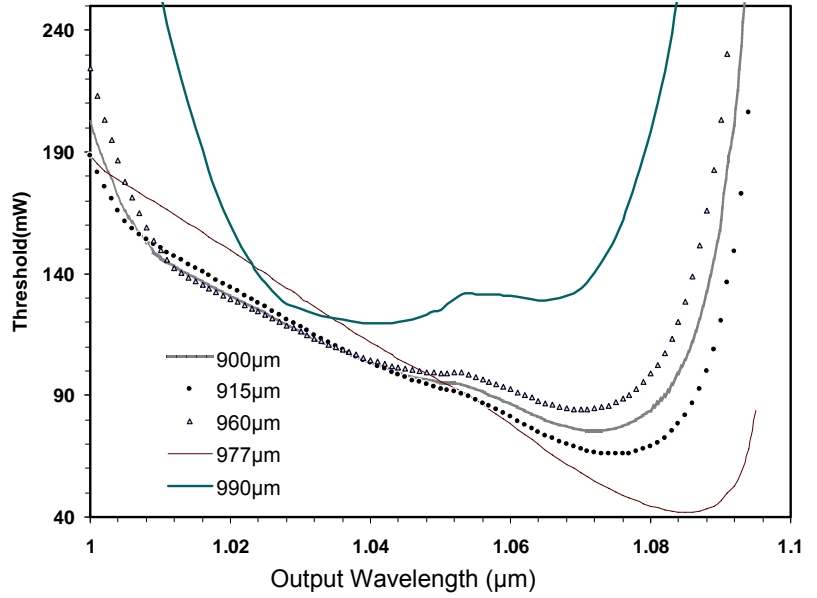

Figure 12. Laser threshold for $2 \mathrm{~m}$ long YDF as a function of operating wavelength.

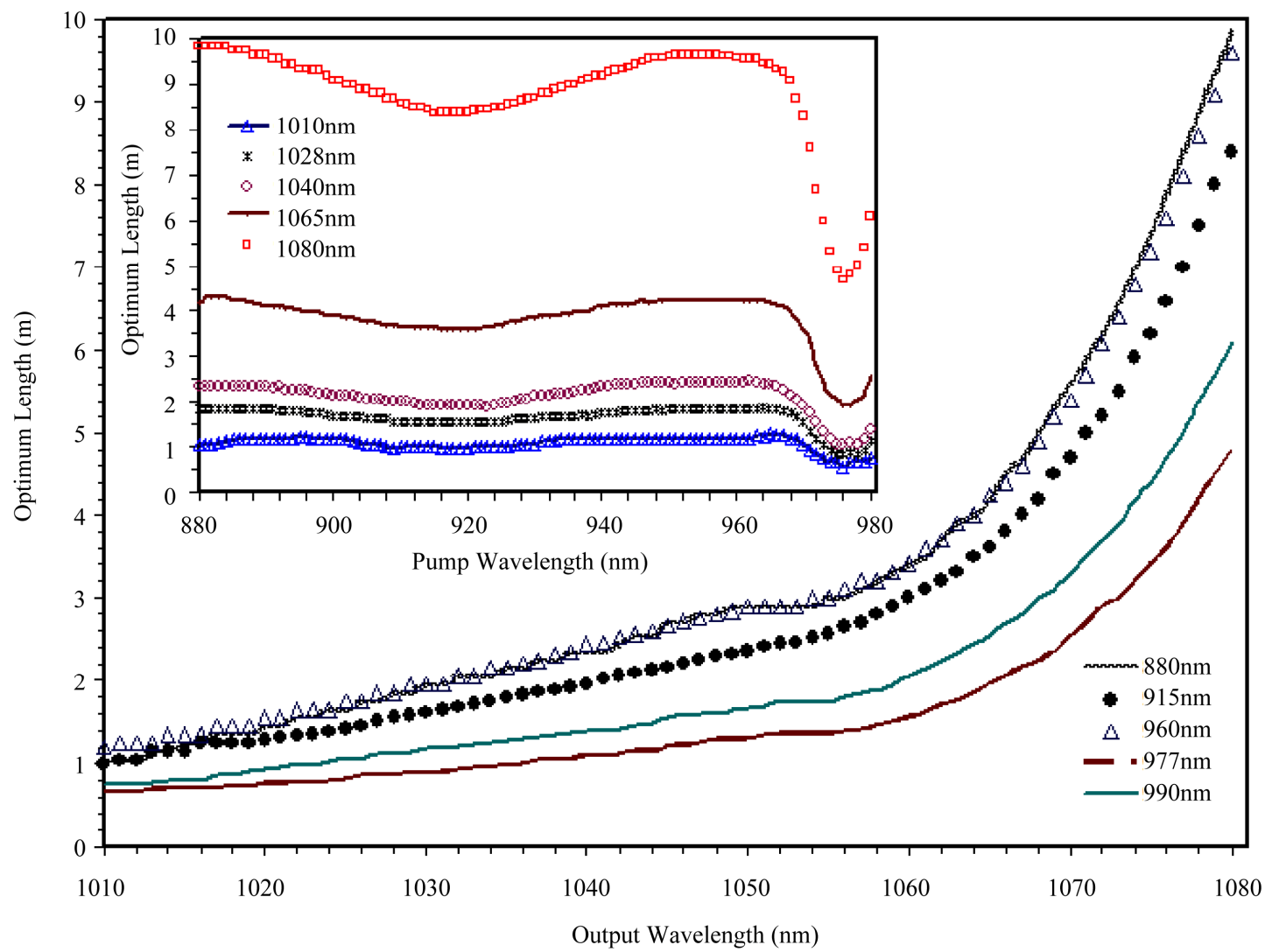

Figure 13. Optimum length as a function of operating wavelength for a fixed input power of $200 \mathrm{~mW}$. Inset shows the dependency of optimum length on the pumping wavelength.

Figure 13 shows the optimum length of proposed YDF laser as a function of operating wavelength when the forward Pump power is fixed at $200 \mathrm{~mW}$. It can be observed from the figure that, the optimum fiber length increases with increasing the pump power. It is clear that the efficiency declines when the YDF is longer or shorter than presented lengths.

As shown in the figure the optimal length shortens when operating wavelengths are shorter. As demonstrated in the figure inset, for pumping near $915 \mathrm{~nm}$ or $975 \mathrm{~nm}$, the optimal length is further shortened while, for pumping near $960 \mathrm{~nm}$, longer lengths of the fiber are 
required.

\section{Conclusion}

In this research, the ASE spectrum characteristics for a highly $\mathrm{Yb}^{3+}$ doped fiber at different pump power levels and pump wavelengths were analyzed. Output power, laser threshold and optimum length were also measured and calculated under wide range of operating conditions. It was shown that, the measured ASE bandwidth (FWHM) would be approximately $32 \mathrm{~nm}$ under $975 \mathrm{~nm}$ pumping scheme while it can be reached up to $55 \mathrm{~nm}$ under $967 \mathrm{~nm}$ forward pumping scheme. Then, we proved that, by setting pumping and operating wavelength to certain proper values, one can reduce the threshold power by more than six times. The threshold power is minimum near pumping wavelength of $974 \mathrm{~nm}$ and at operating wavelength of $1080 \mathrm{~nm}$. Finally the optimal YDF length shortens when operating wavelengths are shorter.

\section{REFERENCES}

[1] V. A. Akulov, D. M. Afanasiev, S. A. Babin, D. V. Churkin, S. I. Kablukov, M. A. Rybakov and A. A. Vlasov, "Frequency Tuning and Doubling in Yb-Doped Fiber Lasers," Laser Physics, Vol. 17, No. 2, 2007, pp. 124-129. doi:10.1134/S1054660X07020120

[2] S. Kablukov, E. Dontsova, V. Akulov, A. Vlasov and S. Babin, "Frequency Doubling of Yb-Doped Fiber Laser to 515 nm,” Laser Physics, Vol. 20, No. 2. 2010, pp. 360364. doi:10.1134/S1054660X10040043

[3] A. S. Kurkov, "Oscillation Spectral Range of Yb-Doped Fiber Lasers,” Laser Physics Letters, Vol. 4, No. 2. 2007, pp. 93-102. doi:10.1002/lapl.200610094

[4] M. R. A. Moghaddam, S. Harun, S. Shahi, K. Lim and H. Ahmad, "Comparisons of Multi-Wavelength Oscillations Using Sagnac Loop Mirror and Mach-Zehnder Interferometer for Ytterbium Doped Fiber Lasers,” Laser Physics, Vol. 20, No. 2, 2010, pp. 516-512. doi:10.1134/S1054660X10030138

[5] S. W. Harun, M. C. Paul, M. R. A. Moghaddam, S. Das, R. Sen, A. Dhar, M. Pal, S. K. Bhadra and H. Ahmad, "Diode-Pumped 1028 nm Ytterbium-Doped Fiber Laser with near 90\% Slope Efficiency,” Laser Physics, Vol. 20,
No. 3. 2010, pp. 656-660. doi:10.1134/S1054660X10050051

[6] A. D. Guzman Chavez, A. V. Kir’yanov, Y. O. Barmenkov and N. N. Il'ichev, "Reversible Photo Darkening and Resonant Photobleaching of Ytterbium Doped Silica Fiber at In-Core $977 \mathrm{~nm}$ and $543 \mathrm{~nm}$ Irradiation," Laser Physics Letters, Vol. 4, No. 10. 2007, pp. 734-739. doi:10.1002/lapl.200710053

[7] L. Xiao, P. Yan, M. Gong, W. Wei and P. Ou, “An Approximate Analytic Solution of Strongly Pumped $\mathrm{Yb}$ Doped Double-Clad Fiber Lasers without Neglecting the Scattering Loss,” Optics Communications, Vol. 230, No. 4-6, 2004, pp. 401-410. doi:10.1016/j.optcom.2003.11.017

[8] Z. Lali-Dastjerdi, F. Kroushavi and M. H. Rahmani, “An Efficient Shooting Method for Fiber Amplifiers and Lasers," Optics \& Laser Technology, Vol. 40, No. 8. 2008, pp. 1041-1046. doi:10.1016/j.optlastec.2008.02.006

[9] D. Xue, Q. Lou and J. Zhou, “Comparison of Yb-Doped Fiber Laser with One-End and Double-End Pumping Configuration," Optics \& Laser Technology, Vol. 39, No. 4, 2007, pp. 871-874. doi:10.1016/j.optlastec.2005.12.005

[10] J. Koponen, M. Söderlund, H. J. Hoffman, D. A. V. Kliner, J. P. Koplow and M. Hotoleanu, "Photodarkening Rate in Yb-Doped Silica Fibers," Applied Optics, Vol. 47, No. 9. 2008, pp. 1247-1256. doi:10.1364/AO.47.001247

[11] G. Demirkhanyan, "Intensities of Inter-Stark Transitions in YAG-Yb ${ }^{3+}$ Crystals,” Laser Physics, Vol. 16, No. 7. 2006, pp. 1054-1057. doi:10.1134/S1054660X0607005X

[12] M. Javadi-Dashcasan, F. Hajiesmaeilbaigi, H. Razzaghi, M. Mahdizadeh and M. Moghadam, "Optimizing the Yb: YAG Thin Disc Laser Design Parameters,” Optics Communications, Vol. 281, No. 18. 2008, pp. 4753-4757. doi:10.1016/j.optcom.2008.05.055

[13] T. W. Huang and W. P. Lin, "Braodband Tunable 1060 nm Fiber Ring Laser Band on Hybrid Amplifier with 45 nm Tuning Range," IEEE Conference on Cross Strait Quad-Regional Radio Science and Wireless Technology, Harbin, 26-30 July 2011, pp. 270-273.

[14] I. Kelson and A. Hardy, "Optimization of Strongly Pumped Fiber Lasers,” Journal of Lightwave Technology, Vol. 17, No. 5, 1999, pp. 891-897. doi:10.1109/50.762908

[15] P. Nandi and G. Jose, "Ytterbium-Doped $\mathrm{P}_{2} \mathrm{O}_{5}-\mathrm{TeO}_{2}$ Glass for Laser Applications,” IEEE Journal of Quantum Electronics, Vol. 42, No. 11. 2006, pp. 115-1121. doi:10.1109/JQE.2006.882557 